\title{
A prediction modeling based on SNOT-22 score for endoscopic nasal septoplasty: a retrospective study
}

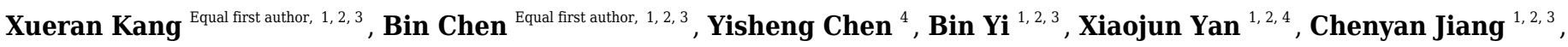 \\ Shulun Wang ${ }^{1,2,3}$, Lixing Lu ${ }^{1,2,3}$, Runjie Shi ${ }^{\text {Corresp. } 1,2,3}$ \\ ${ }^{1}$ Department of Otorhinolaryngology Head and Neck Surgery, Shanghai ninth people's Hospital, Shanghai Jiao Tong University School of Medicine, \\ Shanghai, China \\ 2 Ear Institute, Shanghai JiaoTong University School of Medicine, Shanghai, China \\ 3 Shanghai Key Laboratory of Translational Medicine on Ear and Nose diseases, Shanghai, China \\ 4 Department of Orthopedics, Shanghai General Hospital, Shanghai Jiao Tong University School of Medicine, Shanghai, China \\ Corresponding Author: Runjie Shi \\ Email address: runjieshi@hotmail.com
}

Background: To create a nomogram prediction model for the efficacy of endoscopic nasal septoplasty, and the likelihood of patient benefiting from the operation. Methods: A retrospective analysis of 155 patients with nasal septum deviation (NSD) was performed to develop a predictive model for the efficacy of endoscopic nasal septoplasty. Quality of life (QoL) data was collected before and after surgery using Sinonasal Outcome Test-22 (SNOT-22) scores to evaluate the surgical outcome. An effective surgical outcome was defined as a SNOT-22 score change $\geq 9$ points after surgery. Multivariate logistic regression analysis was then used to establish a predictive model for the NSD treatment. The predictive quality and clinical utility of the predictive model were assessed by C-index, calibration plots, and decision curve analysis. Results: The identified risk factors for inclusion in the predictive model were included. The model had a good predictive power, with a AUC of 0.920 in the training group and a $C$ index of 0.911 in the overall sample. Decision curve analysis revealed that the prediction model had a good clinical applicability. Conclusions: Our prediction model is efficient in predicting the efficacy of endoscopic surgery for NSD through evaluation of factors including: history of nasal surgery, preoperative SNOT-22 score, sinusitis, middle turbinate plasty, BMI, smoking, follow-up time, seasonal allergies, and advanced age. Therefore, it can be cost-effective for individualized preoperative assessment. 
1 A prediction modeling based on SNOT-22 score for endoscopic nasal

2 septoplasty: a retrospective study

3 Running title: A prediction model for nasal septoplasty

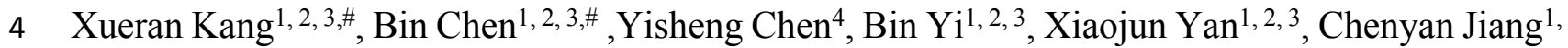

$5 \quad 2,3$, Shulun Wang ${ }^{1,2,3}$, Lixing Lu ${ }^{1,2,3}$, Runjie Shi ${ }^{1,2,3, *}$

$6 \quad{ }^{1}$ Department of Otorhinolaryngology Head and Neck Surgery, Shanghai ninth people's Hospital,

7 Shanghai Jiao Tong University School of Medicine, Shanghai, China

$8 \quad{ }^{2}$ Ear Institute, Shanghai JiaoTong University School of Medicine, Shanghai, China

$9 \quad{ }^{3}$ Shanghai Key Laboratory of Translational Medicine on Ear and Nose diseases, Shanghai, China

${ }^{4}$ Department of Orthopedics, Shanghai General Hospital, Shanghai Jiao Tong University School

of Medicine, Shanghai, China

\#These authors contributed equally to this work.

\section{*Corresponding Author}

Runjie Shi

Department of Otorhinolaryngology Head and Neck Surgery, Shanghai ninth people's Hospital,

JiaoTong University School of Medicine, Shanghai, China; Shanghai Key Laboratory of Bureau Road, Huangpu District, Shanghai 200011, China.

Email: runjieshi@hotmail.com 
23 Tel: +8618019790527 


\section{ABSTRACT}

Background: To create a nomogram prediction model for the efficacy of endoscopic nasal septoplasty, and the likelihood of patient benefiting from the operation.

Methods: A retrospective analysis of 155 patients with nasal septum deviation (NSD) was performed to develop a predictive model for the efficacy of endoscopic nasal septoplasty. Quality of life (QoL) data was collected before and after surgery using Sinonasal Outcome Test-22 (SNOT-22) scores to evaluate the surgical outcome. An effective surgical outcome was defined as a SNOT-22 score change $\geq 9$ points after surgery. Multivariate logistic regression analysis was then used to establish a predictive model for the NSD treatment. The predictive quality and clinical utility of the predictive model were assessed by $\mathrm{C}$-index, calibration plots, and decision curve analysis.

Results: The identified risk factors for inclusion in the predictive model were included. The model had a good predictive power, with a AUC of 0.920 in the training group and a C index of 0.911 in the overall sample. Decision curve analysis revealed that the prediction model had a good clinical applicability.

Conclusions: Our prediction model is efficient in predicting the efficacy of endoscopic surgery for NSD through evaluation of factors including: history of nasal surgery, preoperative SNOT-22 score, sinusitis, middle turbinate plasty, BMI, smoking, follow-up time, seasonal allergies, and advanced age. Therefore, it can be cost-effective for individualized preoperative assessment.

Key words: septoplasty; nasal septum deviation (NSD); nomogram prediction model; quality of 
44 life; SNOT-22 score. 
 \\ INTRODUCTION}

46

47

Nasal septum deviation (NSD) is one of the most frequently encountered diseases in the rhinology clinic in which the nasal septum deviates from the midline, causing the nasal cavity to shrink in size (Cho et al., 2010; MattosWoodard \& Payne, 2011). It occurs in $77-90 \%$ of the general population (Gray, 1978; Mladina et al., 2008). Common symptoms are stuffy nose, headache, nosebleed, etc. NSD is an expensive medical condition, and also acts as a predisposing factor for other diseases (Bousquet et al., 2008; Fokkens et al., 2012). For instance, long-term NSD may indirectly increase the risk of cardiovascular disease (Ozkececi et al., 2016; Uluyol et al., 2016). Conservative treatments for NSD have a low benefit likelihood (Lindsay, 2012; Rhee et al., 2014; Rudy \& Most, 2017), and according to studies published in Lancet in 2019, nasal septoplasty is more effective than non-surgical treatment in adults with NSD (van Egmond et al., 2019). Therefore, in NSD treatment, surgical method should be considered over conservative non-surgical method (Aydogdu et al., 2019). The use of endoscopy provides better illumination for nasal septum correction, making it easier to manage spur or crest. Compared with traditional surgical methods, endoscopic nasal septoplasty surgery has fewer complications and better results. Since it was first described by Stammberger in 1991, it has been increasingly adopted by clinicians and patients (Dell'Aversana Orabona et al., 2018). However, the surgical outcome of NSD correction is affected by many factors including: disease-related factors (such as the severity of the disease, combined with sinusitis and middle turbinate plasty); treatment-related factors (such as surgery and rehabilitation protocol, follow-up time, and other medical-related medications); and patient-related factors (such as gender, BMI, 
65 age, smoking, drinking history, and seasonal allergies) (Ahn et al., 2016; Alakarppa et al., 2018;

66 Becker et al., 2008; Hong et al., 2015). In addition, studies have shown that nasal valve collapse

67 and sinusitis, caused by a history of nasal surgery, could make a profound impact on the efficacy

68 of NSD surgery (Becker et al., 2008; Bhattacharyya, 2005).

69 Despite a number of variables that influence the efficacy of NSD surgery being identified in previous studies, no systematic assessment aimed at predicting the surgery's efficacy exists. Therefore, accurate predictive tools and early individualized interventions could be effective in improving the surgical outcomes in patients (van Egmond et al., 2015). SNOT-22 is deemed a credible, valid and responsive disease-specific instrument (Dietz de Loos et al., 2013; Hopkins et al., 2009; Morley \& Sharp, 2006). In addition, previous researches support that SNOT-22 is an effective and reliable tool in assessing the results of nasal septal surgery or septorhinoplasty (Aydogdu et al., 2019; BucklandThomas \& Harries, 2003; Poirrier et al., 2013). Creating a prediction model based on SNOT-22 score for endoscopic nasal septoplasty was the main objective of this study (Alakarppa et al., 2017; Alakarppa et al., 2018; van Egmond et al., 2019). We hypothesized that based on the degree of improvement in SNOT-22 scores after nasal septoplasty , an effective nomogram model can be developed and used in predicting the likelihood of a benefit outcome from the surgery. clinicians for the efficacy prediction of nasal septoplasty. 
84 MATERIALS \& METHODS

85

86

87

\section{Research object}

This was a retrospective study conducted to establish the efficacy of nasal septoplasty. The study was approved by the Ethics Committee of the Ninth People's Hospital affiliated to Shanghai Jiao Tong University Medical School (approval no. 2017-323-T243), and met the requirements of the Helsinki Declaration. Eligible patients had all been diagnosed with NSD after an electronicnasopharyngoscopy and maxillofacial CT scan between January 2015 and September 2019, at the Department of Otorhinolaryngology, Shanghai Ninth People's Hospital. All the patients were residents of China and had undergone an endoscopic nasal septoplasty, conducted by the same surgeon. Combined with preoperative medical information, we conducted further questionnaire surveys and postoperative follow-ups from February to September 2019 by telephone appointment, outpatient and community follow-up. Prior to the study, we obtained signed informed consent forms from the participating patients. Patients enrolled in the study underwent SNOT-22 scores before and after surgery, and all questionnaires and follow-up were successfully completed. In addition, records of basic clinical characteristics such as age, BMI, etc., were taken from the participants. Difference in SNOT-22 score before and after surgery exceeding 9 points is deemed an effective surgical outcome (Alakarppa et al., 2017).

\section{Subject exclusion criteria}

All patients attending these clinics completed the Sino Nasal Outcome Test (SNOT-22). The following criteria were set for excluding subjects from the study: Patients who had contracted nasal 
104

105

106

107

108

109

110

111

112

113

114

115

116

117

118

119

120

121

122

123

124

tumors diseases or nasal septal perforation; lack of cooperation in completing basic diagnostic tests; fever or infection of unknown origin; and acute heart failure, renal failure or any other organ failure. Among the screened patients, a total of 155 patients met the inclusion criteria were included in the study,including 109 males, aged (14-66 years) with a mean age of (35.3 \pm 13.5$)$ years, and 46 females, aged (17-62 years) with a mean age of $(36.7 \pm 12.1)$ years. We conducted a retrospective analysis on these 155 patients.

\section{Determination of the curative effect of nasal septoplasty}

Sinonasal Outcome Test-22 (SNOT-22) was used to evaluate the disease-specific QoL scores. SNOT-22 is an effective and reliable tool for patients with NSD (Buckland et al., 2003; Dietz de Loos et al., 2013; Hopkins et al., 2009; Hytonen et al., 2012; Morley \& Sharp, 2006; Poirrier et al., 2013). Each question has 0-5 points, and all questions are summed to give a total score between 0 and 110. The higher the SNOT-22 score, the worse the patient's QoL. Postoperative SNOT-22 score change $\geq 9$ points (Alakarppa et al., 2018; Hopkins et al., 2009) was defined as an effective QoL outcome. In this study used the Chinese scale of the SNOT score(Cao et al., 2017).

\section{Medical history and basic data collection}

Subjects responded to self-administered questionnaires on the following characteristics: age, gender, smoking, alcohol status, and chronic disease, history of seasonal allergy symptoms, family history, preoperative nasal steroid use history, history of nasal surgery, nasal bone fracture history, follow-up time etc. Medical history data was reviewed to establish whether the subjects had the following medical conditions; nasal polyps, sinusitis, epistaxis, curved/angulated deviation, and 
125

126

127

128

spur or crest. Besides the nasal septoplasty, we reviewed whether the subjects had undergone a simultaneous operation such as the middle turbinoplasty, inferior turbinoplasty, augmentation rhinoplasty, RFVR of the inferior turbinate,nasal bone fracture reduction, endoscopic sinus surgery. And There are many types of morphological classification methods for nasal septal deviation. In this study, we employed the method proposed by Hong-Ryul Jin to classify the nasal septal deviation into four types (JinLee \& Jung, 2007). The patient's length of hospital stay (day), follow-up period and other data were collected too. The patients were assisted by a physician to complete the SNOT-22 score.

\section{Statistical Analysis}

155 selected patients were randomly divided into training and validation (7:3) group for diagnostic and prognostic analysis and used for evaluation of the model. Statistical analysis was performed by R software (version 3.5.3). All pre-determined factors were included in the least absolute shrinkage and selection operator (LASSO) analysis data reduction, and screening of appropriate predictors (FriedmanHastie \& Tibshirani, 2010; Kidd et al., 2018; SauerbreiRoyston \& Binder, 2007). In the Lasso model, a five-fold cross-validation approach was used for the choice of optimal parameters(Le \& Nguyen, 2019; LeYapp \& Yeh, 2019b). These features were further filtered using a Support Vector Machine-Recursive Feature Elimination (SVM-RFE) algorithm. Support vector machines are increasingly used in the field of bioinformatics(Le, 2019; Le et al., 2019a). The aim of this method was to find an optimal plane in the multidimensional space, which could divide all sample units into two classes, and also maximize the distance between the two nearest points in the different classes. The edge point between the two closest points is known as 
146 the SVM, and the split hyperplane is found in the space between them. The SVM was then used

147 as a classifier for the SVM-RFE algorithm, from the most relevant to the least relevant ordering of

148 the features. The SVM-RFE algorithm may be superior to the linear discriminant analysis, and the

149 mean square error method in selecting relevant features and removing redundant features, 150 especially in the case of a small number of samples (Huang et al., 2014b).

151 A predictive model was then built using a multivariate logistic regression model. Based on 152 the collected patient data, we established a predictive model including all the best predictors, to 153 predict the outcome of surgical correction in patients with NSD (Balachandran et al., 2015; Iasonos 154 et al., 2008). We plotted the calibration curve to evaluate the nomogram's accuracy (Kramer \& Zimmerman, 2007). To further quantify the discrimination performance of the nomogram, we measured the c-index and the AUC. R language package was then used to perform further iterations 157 (10,000 repetitive samples) on the nomogram to calculate a more accurate C-index (Pencina \& D'Agostino, 2004). In addition, we also used an external dataset to corroborate our results. By quantifying the net benefit of different threshold probabilities in patient information, decision curve analysis was used to assess the clinical utility of the nomogram. All statistical tests were bilateral and statistical significance was set at $\mathrm{P}<0.05$. This study was conducted in line with the guidelines (Collins et al., 2015).

RESULTS 
patients) and unimproved group (28 patients) according to the changes in SNOT-22 scores before and after surgery. Table 1 outlines the overall characteristics of the patients data.

\section{Screening prediction}

We applied the LASSO regression model (Fig.1A,B) to reduce the factors investigated in this

study from 48 to 20. On the basis of Harrell's guidelines, when the outcome is binary, the minimum

value of the frequencies of the two response levels should be greater than 10 times the number of

predictors (Harrell, 2001; Iasonos et al., 2008). As shown in Figures 1a and b in the article, simple

use of lasso analysis requires the inclusion of 20 variables in the model. If some variables are not

filtered and excluded, it will require that at least 200 samples be included for training cohort, which

is difficult to achieve. Nevertheless, we have collected all possible data and added new external

data. To address this problem, we screened the factors further using the SVM-RFE algorithm to

obtain "the least characteristic factor" (table. 2). The SVM-RFE algorithm may be superior to the

linear discriminant analysis and the mean square error method in selecting relevant features and

excluding redundant features, especially in the case of a small number of samples (Huang et al.,

2014a). Finally, it proves that the prediction model established by the characteristic factors

screened by svm has higher accuracy. The best prediction model had nine optimal features with

an average cross-validation score of 0.8681 (Fig.1C). These characteristics included history of

nasal surgery, preoperative SNOT-22 score, sinusitis, middle turbinate plasty, BMI, smoking, 
187

188

190

191

192

193

194

195

196

197

198

199

200

201

202

203

204

205

206

207

confirm the performance of the nomogram in predicting the efficacy and clinical utility of NSD

correction. As the sample size increases, the composition of optimal characteristic factors may also

change, and at that time we will have the conditions necessary to further evaluate which method

is more effective in establishing a prediction model.

\section{Building a personalized predictive model}

After logistic model analysis of the nine predictors (table. 3), we used $\mathrm{R}$ software nomogramEx package to construct the nomogram, as shown in Figure 2. Among them, age, SNOT-22 score, history of sinusitis and nasal surgery were revealed as significant factors affecting the efficacy of nasal septum surgery $(\mathrm{P}<0.05)$.

\section{Inspection of the nomogram prediction model}

The calibration curve of nomogram was consistent (Fig. 3A), indicating that the model was competent to predict the surgical outcome of NSD. The model had a good predictive power, with a C-index of 0.920 (95\% confidence interval:0.854-0.986), 0.920 (95\% confidence interval:0.8540.986), 0.834 (95\% confidence interval: $0.655-1.000)$ and 0.765 (95\% confidence interval: $0.555-$ 0.974) in the training queue, the validation queue, the overall sample, and the external dataset, respectively. In addition, the AUC of the prediction model were 0.920 in training set (the AUC curve is shown in Fig.3B). For our research, we believe that increasing the sample size will be the main solution to overfitting. We have tested the out-of-group samples according to your suggestions and still obtain a high accuracy (Figure 3B). Therefore, we believe that using larger sample size in future research will greatly decrease the risk of overfitting.

\section{Clinical application}


212 et al., 2018). Within this range, net benefit was comparable with several overlaps, on the basis of

213 the prediction nomogram. Therefore, the decision curve showed that clinical decisions based on

214 the nomogram prediction model yielded better returns. This means that the current predictive model can achieve better clinical practice through early planning of clinical interventions and better prediction of surgical outcomes, therefore ensuring administration of the most suitable 217 treatment.

\section{DISCUSSION}

In this retrospective study, we established a continuum prediction model for surgical

outcomes based on nine factors. The nomogram prediction model has good quantitative indicators

and is suitable for prognosis and evaluation of clinical outcomes (Wei et al., 2017). To the best of

our knowledge, this study is the first to apply a nomogram model to the evaluation of the efficacy

of endoscopic NSD correction. A previous study validated the usefulness of the SNOT-22 score in patients with nasal septum deviation, but did not construct a predictive model based on the

SNOT-22 score (PannuChadha \& Kaur, 2009). Early identification of patients with nasal septum 
227 resources, and provide reasonable prediction of the surgical effects by doctors and patients. This

228 will improve the doctor-patient relationship.

229 We created and validated a new tool for predicting the efficacy of endoscopic NSD correction.

230 The tool uses nine readily available variables and has a high predictive accuracy. The incorporation

231 of various characteristic factors into an easy-to-use nomogram facilitates individualized evaluation

232 of the efficacy of endoscopic NSD correction. Our internal sampling showed the model's strong

233 predictive ability. In addition, the high c-index and AUC index indicate that this predictive model

234 can be widely and accurately used for the evaluation of therapeutic effect of nasal endoscopic

235 deviation (Wei et al., 2017).

236 Studies that compare nasal patency before and after surgery(Cantone et al., 2018; Hsu et al.,

237 2017) have been reported to have a higher risk of bias since treatment may improve the quality of

238 life of patients, and be reflected in the SNOT-22 score. Therefore, in this study we selected SNOT-

23922 score change as the primary outcome since it included quality of life for both general and

240 specific diseases (van Egmond et al., 2019). Moreover, assessment of postoperative quality of life

241 in patients facilitates interdisciplinary research, and makes it possible to compare the depth of nasal

242 septum to other interventions.

243 In this study, $81.9 \%$ of the patients attained satisfactory results after NSD surgery. In the risk

244 factor analysis, history of nasal surgery, lower preoperative SNOT-22 score, combined sinusitis,

245 combined with middle turbinate plasty, high BMI, smoking, longer follow-up, and advanced age

246 were revealed to be the poor risk factors for NSD outcomes. Among these, SNOT-22 score, history

247 of nasal surgery, sinusitis and age were the main factors affecting the efficacy of nasal septum 
248

249

250

251

252

253

254

255

256

257

258

259

260

261

262

263

264

265

266

267

268

surgery.

Our results suggest that preoperative SNOT-22 score is a good predictor of surgical outcomes.

By definition, a SNOT-22 score change of 9 points after surgery is considered a good surgical

outcome, a change that cannot be attained by patients with a lower preoperative SNOT-22 score.

Therefore, the severity of a patient's condition has a bearing on the SNOT-22 score change, with

more severe patients likely to record improvement in the quality of life after surgery. In addition,

studies have shown that a preoperative SNOT-22 score of $\geq 20$ can significantly improve the

likelihood of surgical outcomes (Hopkins et al., 2009). The history of nasal surgery is a risk factor

for the prognosis of surgical outcomes, since repeated nasal surgery often results into more complex lesions of local tissue, other than just a scar formation. We found that the probability of a nasal valve collapse during revision surgery was higher, and could be a likely cause of refractory nasal congestion (Becker et al., 2008), making efficacy of surgery in such patients worse.

Similar to most studies, we identified sinusitis as one of the risk factors for poor outcome in NSD surgery. NSD patients with CRS have been reported to have poor prognosis, longer treatment cycles, and higher consumption of medical resources (Ahn et al., 2016; Bhattacharyya, 2005). In addition, NSD also acts as a susceptibility factor for sinusitis by reducing nasal mucociliary activity, and inducing chronic inflammation and squamous metaplasia (Ahn et al., 2016; JiFu \& Song, 2015; Kamani et al., 2014; Karatas et al., 2015). Therefore, sinusitis treatment is a serious problem in the clinical treatment of NSD. Moreover, studies have shown that in patients with refractory sinusitis, no significant difference is observed between the efficacy of nasal septoplasty and conservative treatment methods (Rudmik et al., 2011). Our predictive model revealed that the 
269

270

271

272

273

274

275

276

277

278

279

280

281

282

283

284

285

286

287

288

289

middle turbinate plasty could affect the outcome of surgical treatment, making it a potential independent risk factor for sinusitis (Javadrashid et al., 2014). Concha bullosa, which is the aberrant pneumatization of the middle turbinate, affects the shape of the nasal septum leading to the occurrence of NSD (Lee et al., 2008). Incidence of Concha bullosa in patients with NSD has been shown to be higher in previous studies (Yazici, 2019). Patients with both nasal septum and Concha bullosa often display more severe histomorphological variation (Tomblinson et al., 2016).

Subjective scoring for improvement in nasal symptoms and changes in short-term quality of life are often influenced by surgical comfort effects, hence should be performed early in the postoperative period. In our findings, we observed that patients with nasal septum surgery became less aggressive with prolonged postoperative time (Bitzer, 2004; BitzerDorning \& Schwartz, 1996; JessenIvarsson \& Malm, 1989; Konstantinidis et al., 2005).

In addition, over time, uncontrolled chronic sinusitis or other nasal structural lesions could affect the patient's subjective perception of nasal septoplasty, leading to a bias surgical evaluation (Jessen et al., 1989; StewartRobinson \& Wilson, 1996). Notably, the prognosis of smokers deteriorated faster than non-smokers. Studies have shown that after nasal surgery, smokers record longer recovery time due to slow wound healing process (CetinerCavusoglu \& Duzer, 2017). In addition, smoking also leads to a decrease in nasal mucociliary clearance (MCC) time (Karaman \& Tek, 2009). This indicates that smoking could be a potential risk factor for poor prognosis in NSD surgery.

Recent studies have reported that among children and adolescents with severe symptoms, nasal septoplasty is a safe and effective procedure (FullerLevesque \& Lindsay, 2018). However, 
290

291

292

293

294

295

296

297

298

299

300

301

302

303

304

305

306

307

308

309

310

patient satisfaction significantly declined with increase in age (Habesoglu et al., 2015). In addition, our multivariate analysis model identified advanced age as a risk factor for poor outcome after NSD (Ahn et al., 2016). Notably, the improvement of airway ventilation after NSD was more significant in young people, with the older patients not benefiting significantly after surgery (Ratajczak et al., 2009). Patients with NSD have poor nasal ventilation (Glotov et al., 2017), and obesity can aggravate the condition (Ertugay et al., 2015). Reports have shown that patients with a history of seasonal allergies in the nasal cavity have a better prognosis. This could be attributed to the poor adaptation of the nasal microenvironment to environmental changes in patients with severe NSD. However, the situation has been shown to be reversible though NSD surgery.

When considering surgery, clinical benefits, morbidity and associated complications, should be taken into consideration by patients and physicians. Therefore, an accurate prognosis would enhance doctors' assessment for the likelihood of patient's benefit and pre-operative communication with patients. In addition, this minimizes wastage of the costly medical resources. Therefore, in this study, we have developed an effective predictive model for the efficacy of NSD, which provides further theoretical guidance for clinical surgical treatment, and research of NSD.

Despite the efficiency of our model, accurate determination of patient's benefit after surgery requires reasonable evaluation, and targeted interventions aimed at improving the postoperative efficacy of patients with NSD. To eliminate bias that could arise from differences in surgical proficiency between surgeons, all the operations were performed by an experienced surgeon. However, our study had the following limitations. First, the proportion of females in our sample was lower than males due to the higher risk of NSD in the latter. In addition, this study only focused 
311

312 313

314 315 316 317 318 319

on patients from China therefore does not represent patients with NSD in other countries and regions. Second, not all factors affecting the efficacy of endoscopic nasal septoplasty were included in the risk factor analysis. It is challenging to incorporate all objective factors in the statistical analysis. This is one of the dilemmas inherent in machine learning. We have attempted to obtain all the characteristic factors that we could reasonably access. Our results show that the factors obtained are representative, and they can effectively describe the prognosis of patients. Third, although our nomogram prediction model exhibited good prediction accuracy, its further optimization is required using more data. And SNOT-22 version is not yet fully content valid for NSD patients because other nasal symptoms common to NSD patients, such as epistaxis, which we added into our questionnaire, are not included in the current SNOT-22 architecture. Therefore, previous study suggests that further studies be performed to improve the SNOT score (Leong \& Webb, 2018).

\section{CONCLUSIONS}

In this study, we developed a robust predictive model that can be used by clinicians for the efficacy prediction of endoscopic NSD correction. Our model incorporates the history of nasal surgery, lower preoperative SNOT-22 score, combined sinusitis, combined with middle turbinate plasty, high BMI, smoking, longer follow-up, and advanced risk factors. We demonstrate that the use of this model for the prediction of the therapeutic effect of nasal endoscopic deviation, is effective and economical. In addition, ability of the clinicians to estimate individual risk ensures better communication with patients regarding the best treatment option. However, further research 
331 should be conducted to confirm the efficiency of this nomogram prediction model in predicting

332 the efficacy and clinical utility of NSD correction.

\section{ADDITIONAL INFORMATION AND DECLARATIONS}

334 Acknowledgements: Thanks for the anonymous reviewers for their valuable comments and 335 suggestions that helped improve the quality of our manuscript.

336 Funding: This work was supported by Natural Science Foundation of Shanghai grants 337 (14ZR14233800) and Ninth Hospital Clinical Research Booster Program (JYL028).

\section{Competing interests}

339 The authors declare that they have no competing interests.

340 Ethics approval and consent to participate

341 This study was approved by the Institutional Ethics Review Board of Ninth People's Hospital

342 affiliated to Shanghai Jiao Tong University Medical School (approval no. 2017-323-T243).

343 Informed written consent was obtained from the participating patients. 


\section{REFERENCES}

345 Ahn JC, Kim JW, Lee CH, and Rhee CS. 2016. Prevalence and Risk Factors of Chronic 
364

365

366

367

368

369

Balachandran VP, Gonen M, Smith JJ, and DeMatteo RP. 2015. Nomograms in oncology: more than meets the eye. The Lancet Oncology 16:e173-180. 10.1016/s1470-2045(14)71116-7

Becker SS, Dobratz EJ, Stowell N, Barker D, and Park SS. 2008. Revision septoplasty: review of sources of persistent nasal obstruction. American journal of rhinology 22:440-444. 10.2500/ajr.2008.22.3200

Bhattacharyya N. 2005. Symptom and disease severity differences between nasal septal deviation and chronic rhinosinusitis. Otolaryngology--head and neck surgery : official journal of American Academy of Otolaryngology-Head and Neck Surgery 133:173-177. 10.1016/j.otohns.2005.03.082

Bitzer EM. 2004. Nasal surgery: evidence of efficacy. The patient's view on outcomes of septal surgery. Rhinology 42:250-252.

Bitzer EM, Dorning H, and Schwartz FW. 1996. Clinical success of surgical correction of the nasal septum. Laryngo- rhino- otologie 75:649-656; discussion 656-649. 10.1055/s-2007997651

Bousquet J, Khaltaev N, Cruz AA, Denburg J, Fokkens WJ, Togias A, Zuberbier T, BaenaCagnani CE, Canonica GW, van Weel C, Agache I, Ait-Khaled N, Bachert C, Blaiss MS, Bonini S, Boulet LP, Bousquet PJ, Camargos P, Carlsen KH, Chen Y, Custovic A, Dahl R, Demoly P, Douagui H, Durham SR, van Wijk RG, Kalayci O, Kaliner MA, Kim YY, Kowalski ML, Kuna P, Le LT, Lemiere C, Li J, Lockey RF, Mavale-Manuel S, Meltzer EO, Mohammad Y, Mullol J, Naclerio R, O'Hehir RE, Ohta K, Ouedraogo S, Palkonen S, Papadopoulos N, Passalacqua G, Pawankar R, Popov TA, Rabe KF, Rosado-Pinto J, 
Scadding GK, Simons FE, Toskala E, Valovirta E, van Cauwenberge P, Wang DY,

Buckland JR, Thomas S, and Harries PG. 2003. Can the Sino-nasal Outcome Test (SNOT-22) be used as a reliable outcome measure for successful septal surgery? Clinical otolaryngology and allied sciences 28:43-47. 10.1046/j.1365-2273.2003.00663.x

Cantone E, Ricciardiello F, Oliva F, De Corso E, and Iengo M. 2018. Septoplasty: is it possible to identify potential "predictors" of surgical success? Acta otorhinolaryngologica Italica : organo ufficiale della Societa italiana di otorinolaringologia e chirurgia cervico-facciale 38:528-535. 10.14639/0392-100x-2072

Cao C, Yang X, Xiong S, Peng W, Nie M, Liang Q, Feng C, Yi Z, and Lv Y. 2017. Clinical significance of the Chinese version of SNOT-22 in preoperative assessment of posterior paranasal sinus lesions. Chinese Journal of Otorhinolaryngology-Skull Base Surgery:328332. 
406 Cetiner H, Cavusoglu I, and Duzer S. 2017. The effect of smoking on perforation development

407 and healing after septoplasty. American journal of rhinology \& allergy 31:63-65.

408

409

410

411

412

413

414

415

416

417

418

419

420

421

422

423

424

425

426

10.2500/ajra.2017.31.4406

Cho YS, Choi SH, Park KH, Park HJ, Kim JW, Moon IJ, Rhee CS, Kim KS, Sun DI, Lee SH, Koo JW, Koh YW, Lee KH, Lee SW, Oh KW, Pyo EY, Lee A, Kim YT, and Lee CH. 2010. Prevalence of otolaryngologic diseases in South Korea: data from the Korea national health and nutrition examination survey 2008. Clinical and experimental otorhinolaryngology 3:183-193. 10.3342/ceo.2010.3.4.183

Collins GS, Reitsma JB, Altman DG, and Moons KGM. 2015. Transparent Reporting of a Multivariable Prediction Model for Individual Prognosis or Diagnosis (TRIPOD): The TRIPOD Statement. European urology 67:1142-1151. 10.1016/j.eururo.2014.11.025

Dell'Aversana Orabona G, Romano A, Abbate V, Salzano G, Piombino P, Farina F, Pansini A, Iaconetta G, and Califano L. 2018. Effectiveness of endoscopic septoplasty in different types of nasal septal deformities: our experience with NOSE evaluation. Acta otorhinolaryngologica Italica : organo ufficiale della Societa italiana di otorinolaringologia e chirurgia cervico-facciale 38:323-330. 10.14639/0392-100x-1067

Dietz de Loos DA, Segboer CL, Gevorgyan A, and Fokkens WJ. 2013. Disease-specific qualityof-life questionnaires in rhinitis and rhinosinusitis: review and evaluation. Current allergy and asthma reports 13:162-170. 10.1007/s11882-012-0334-8

Ertugay CK, Toros SZ, Karaca CT, Kulekci S, Verim A, Ertugay OC, and Naiboglu B. 2015. Is septoplasty effective on habitual snoring in patients with nasal obstruction? European 
427

428

429

430

431

432

433

434

435

436

437

438

439

440

441

442

443

444

445

446

447

archives of oto-rhino-laryngology : official journal of the European Federation of Oto-

Rhino-Laryngological Societies (EUFOS) : affiliated with the German Society for Oto-

Rhino-Laryngology - Head and Neck Surgery 272:1687-1691. 10.1007/s00405-014-3260-7

Fokkens WJ, Lund VJ, Mullol J, Bachert C, Alobid I, Baroody F, Cohen N, Cervin A, Douglas

R, Gevaert P, Georgalas C, Goossens H, Harvey R, Hellings P, Hopkins C, Jones N, Joos G,

Kalogjera L, Kern B, Kowalski M, Price D, Riechelmann H, Schlosser R, Senior B, Thomas

M, Toskala E, Voegels R, Wang de Y, and Wormald PJ. 2012. EPOS 2012: European

position paper on rhinosinusitis and nasal polyps 2012. A summary for

otorhinolaryngologists. Rhinology 50:1-12. 10.4193/Rhino50E2

Friedman J, Hastie T, and Tibshirani R. 2010. Regularization Paths for Generalized Linear

Models via Coordinate Descent. Journal of statistical software 33:1-22.

Fuller JC, Levesque PA, and Lindsay RW. 2018. Functional septorhinoplasty in the pediatric and adolescent patient. International journal of pediatric otorhinolaryngology 111:97-102.

10.1016/j.ijporl.2018.06.003

Glotov AV, Fedorova TN, Goltyapin VV, and Akhmedov VA. 2017. Nocturnal pulse oximetry indicators in the evaluation of obstructive sleep apnea syndrome in outpatients with concomitant diseases of the upper respiratory tract and overweight. Terapevticheskii arkhiv 89:28-33. 10.17116/terarkh2017891228-33

Gray LP. 1978. Deviated nasal septum. Incidence and etiology. The Annals of otology, rhinology \& laryngology Supplement 87:3-20. 10.1177/00034894780873s201

Habesoglu M, Kilic O, Caypinar B, and Onder S. 2015. Aging as the Impact Factor on 
448

449

450

451

452

453

454

455

456

457

458

459

460

461

462

463

464

465

466

467

468

Septoplasty Success. The Journal of craniofacial surgery 26:e419-422.

$10.1097 /$ scs.0000000000001879

Harrell FJ. 2001. Regression Modeling Strategies With Applications to Linear Models, Logistic Regression, and Survival Analysis. New York, NY, Springer Verlag

Hong SD, Lee NJ, Cho HJ, Jang MS, Jung TY, Kim HY, Chung SK, and Dhong HJ. 2015.

Predictive factors of subjective outcomes after septoplasty with and without turbinoplasty: can individual perceptual differences of the air passage be a main factor? International forum of allergy \& rhinology 5:616-621. 10.1002/alr.21508

Hopkins C, Gillett S, Slack R, Lund VJ, and Browne JP. 2009. Psychometric validity of the 22item Sinonasal Outcome Test. Clinical otolaryngology : official journal of ENT-UK ; official journal of Netherlands Society for Oto-Rhino-Laryngology \& Cervico-Facial Surgery 34:447-454. 10.1111/j.1749-4486.2009.01995.x

Hsu HC, Tan CD, Chang CW, Chu CW, Chiu YC, Pan CJ, and Huang HM. 2017. Evaluation of nasal patency by visual analogue scale/nasal obstruction symptom evaluation questionnaires and anterior active rhinomanometry after septoplasty: a retrospective one-year follow-up cohort study. Clinical otolaryngology : official journal of ENT-UK ; official journal of Netherlands Society for Oto-Rhino-Laryngology \& Cervico-Facial Surgery 42:53-59. $10.1111 /$ coa. 12662

Huang ML, Hung YH, Lee WM, Li RK, and Jiang BR. 2014a. SVM-RFE based feature selection and Taguchi parameters optimization for multiclass SVM classifier.

TheScientificWorldJournal 2014:795624. 10.1155/2014/795624 
469 Huang ML, Hung YH, Lee WM, Li RK, and Jiang BR. 2014b. SVM-RFE based feature

470 selection and Taguchi parameters optimization for multiclass SVM classifier. The Scientific World Journal 2014:795624. 10.1155/2014/795624

472

473

474

475

476

477

478

479

480

481

482

483

484

485

486

487

488

489

Hytonen ML, Lilja M, Makitie AA, Sintonen H, and Roine RP. 2012. Does septoplasty enhance the quality of life in patients? European archives of oto-rhino-laryngology : official journal of the European Federation of Oto-Rhino-Laryngological Societies (EUFOS) : affiliated with the German Society for Oto-Rhino-Laryngology - Head and Neck Surgery 269:24972503. $10.1007 / \mathrm{s} 00405-012-1931-9$

Iasonos A, Schrag D, Raj GV, and Panageas KS. 2008. How to build and interpret a nomogram for cancer prognosis. Journal of clinical oncology : official journal of the American Society of Clinical Oncology 26:1364-1370. 10.1200/jco.2007.12.9791

Javadrashid R, Naderpour M, Asghari S, Fouladi DF, and Ghojazadeh M. 2014. Concha bullosa, nasal septal deviation and paranasal sinusitis; a computed tomographic evaluation. B-ent 10:291-298.

Jessen M, Ivarsson A, and Malm L. 1989. Nasal airway resistance and symptoms after functional septoplasty: comparison of findings at 9 months and 9 years. Clinical otolaryngology and allied sciences 14:231-234. 10.1111/j.1365-2273.1989.tb00366.x

Ji X, Fu H, and Song A. 2015. [Study on the correlation between chronic sinusitis with nasal septum deviation]. Lin chuang er bi yan hou tou jing wai ke za zhi = Journal of clinical otorhinolaryngology, head, and neck surgery 29:1103-1104.

Jin HR, Lee JY, and Jung WJ. 2007. New Description Method and Classification System for 
490

491

492

493

494

495

496

497

498

499

500

501

502

503

504

505

506

507

508

509

510

Septal Deviation.

Kamani T, Yilmaz T, Surucu S, Bajin MD, Gunaydin RO, and Kuscu O. 2014. Histopathological changes in nasal mucosa with nasal septum deviation. European archives of oto-rhinolaryngology : official journal of the European Federation of Oto-Rhino-Laryngological Societies (EUFOS) : affiliated with the German Society for Oto-Rhino-Laryngology - Head and Neck Surgery 271:2969-2974. 10.1007/s00405-014-2990-x

Karaman M, and Tek A. 2009. Deleterious effect of smoking and nasal septal deviation on mucociliary clearance and improvement after septoplasty. American journal of rhinology \& allergy 23:2-7. 10.2500/ajra.2009.23.3253

Karatas D, Koc A, Yuksel F, Dogan M, Bayram A, and Cihan MC. 2015. The Effect of Nasal Septal Deviation on Frontal and Maxillary Sinus Volumes and Development of Sinusitis. The Journal of craniofacial surgery 26:1508-1512. 10.1097/scs.0000000000001809

Kidd AC, McGettrick M, Tsim S, Halligan DL, Bylesjo M, and Blyth KG. 2018. Survival prediction in mesothelioma using a scalable Lasso regression model: instructions for use and initial performance using clinical predictors. BMJ open respiratory research 5:e000240. 10.1136/bmjresp-2017-000240

Konstantinidis I, Triaridis S, Triaridis A, Karagiannidis K, and Kontzoglou G. 2005. Long term results following nasal septal surgery. Focus on patients' satisfaction. Auris, nasus, larynx 32:369-374. 10.1016/j.anl.2005.05.011

Kramer AA, and Zimmerman JE. 2007. Assessing the calibration of mortality benchmarks in critical care: The Hosmer-Lemeshow test revisited. Critical care medicine 35:2052-2056. 
512

513

514

515

516

517

518

519

520

521

522

523

524

525

526

527

528

529

530

531

Le NQ, and Nguyen BP. 2019. Prediction of FMN Binding Sites in Electron Transport Chains based on 2-D CNN and PSSM Profiles. IEEE/ACM transactions on computational biology and bioinformatics. 10.1109/tcbb.2019.2932416

Le NQK. 2019. iN6-methylat (5-step): identifying DNA N(6)-methyladenine sites in rice genome using continuous bag of nucleobases via Chou's 5-step rule. Molecular genetics and genomics : $M G G$ 294:1173-1182. 10.1007/s00438-019-01570-y

Le NQK, Yapp EKY, Ho QT, Nagasundaram N, Ou YY, and Yeh HY. 2019a. iEnhancer-5Step: Identifying enhancers using hidden information of DNA sequences via Chou's 5-step rule and word embedding. Analytical biochemistry 571:53-61. 10.1016/j.ab.2019.02.017

Le NQK, Yapp EKY, and Yeh HY. 2019b. ET-GRU: using multi-layer gated recurrent units to identify electron transport proteins. BMC bioinformatics 20:377. 10.1186/s12859-019-29725

Lee JS, Ko IJ, Kang HD, and Lee HS. 2008. Massive concha bullosa with secondary maxillary sinusitis. Clinical and experimental otorhinolaryngology 1:221-223.

$10.3342 /$ ceo.2008.1.4.221

Leong SC, and Webb CJ. 2018. Sino-Nasal Outcome Test-22 quality-of-life patterns in patients presenting with nasal septal perforation. Clinical otolaryngology : official journal of ENTUK ; official journal of Netherlands Society for Oto-Rhino-Laryngology \& Cervico-Facial Surgery 43:604-608. 10.1111/coa.13031 
533

534

535

536

537

538

539

540

541

542

543

544

545

546

547

548

549

550

551

552

Mattos JL, Woodard CR, and Payne SC. 2011. Trends in common rhinologic illnesses: analysis of U.S. healthcare surveys 1995-2007. International forum of allergy \& rhinology 1:3-12. 10.1002/alr.20003

Mladina R, Cujić E, Subarić M, and Vuković K. 2008. Nasal septal deformities in ear, nose, and throat patients: an international study. American journal of otolaryngology 29:75-82. 10.1016/j.amjoto.2007.02.002

Morley AD, and Sharp HR. 2006. A review of sinonasal outcome scoring systems - which is best? Clinical otolaryngology : official journal of ENT-UK ; official journal of Netherlands Society for Oto-Rhino-Laryngology \& Cervico-Facial Surgery 31:103-109. 10.1111/j.17494486.2006.01155.x

Ozkececi G, Akci O, Bucak A, Ulu S, Yalim Z, Aycicek A, Onrat E, and Avsar A. 2016. The effect of septoplasty on pulmonary artery pressure and right ventricular function in nasal septum deviation. European archives of oto-rhino-laryngology: official journal of the European Federation of Oto-Rhino-Laryngological Societies (EUFOS) : affiliated with the German Society for Oto-Rhino-Laryngology - Head and Neck Surgery 273:3747-3752. $10.1007 / \mathrm{s} 00405-016-4042-1$

Pannu KK, Chadha S, and Kaur IP. 2009. Evaluation of benefits of nasal septal surgery on nasal symptoms and general health. Indian journal of otolaryngology and head and neck surgery : official publication of the Association of Otolaryngologists of India 61:59-65. 
553 Pencina MJ, and D'Agostino RB. 2004. Overall C as a measure of discrimination in survival 554 analysis: model specific population value and confidence interval estimation. Statistics in medicine 23:2109-2123. 10.1002/sim.1802

556

557

558

559

560

561

562

563

564

565

566

567

568

569

570

571

572

573

Poirrier AL, Ahluwalia S, Goodson A, Ellis M, Bentley M, and Andrews P. 2013. Is the SinoNasal Outcome Test-22 a suitable evaluation for septorhinoplasty? The Laryngoscope 123:76-81. 10.1002/lary.23615

Ratajczak J, Rapiejko P, Wojdas A, and Jurkiewicz D. 2009. Influence of handicapped of patency nose on quality created of voice. Otolaryngologia polska = The Polish otolaryngology 63:58-63. 10.1016/s0030-6657(09)70191-x

Rhee JS, Sullivan CD, Frank DO, Kimbell JS, and Garcia GJ. 2014. A systematic review of patient-reported nasal obstruction scores: defining normative and symptomatic ranges in surgical patients. JAMA facial plastic surgery 16:219-225; quiz 232. 10.1001/jamafacial.2013.2473

Rudmik L, Mace J, Ferguson BJ, and Smith TL. 2011. Concurrent septoplasty during endoscopic sinus surgery for chronic rhinosinusitis: does it confound outcomes assessment? The Laryngoscope 121:2679-2683. 10.1002/lary.22361

Rudy SF, and Most SP. 2017. Rhinoplasty. Jama 318:1406. 10.1001/jama.2017.13267

Sauerbrei W, Royston P, and Binder H. 2007. Selection of important variables and determination of functional form for continuous predictors in multivariable model building. Statistics in medicine 26:5512-5528. 10.1002/sim.3148

Stewart EJ, Robinson K, and Wilson JA. 1996. Assessment of patient's benefit from rhinoplasty. 
Rhinology 34:57-59.

575 Tomblinson CM, Cheng MR, Lal D, and Hoxworth JM. 2016. The Impact of Middle Turbinate

576 Concha Bullosa on the Severity of Inferior Turbinate Hypertrophy in Patients with a

577

578

579

580

581

582

583

584

585

586

587

588

589

590

591

592

593

594

Peer) reviewing PDF | (2020:03:46884:1:1:NEW 28 Jul 2020) 
595 Wei L, Champman S, Li X, Li X, Li S, Chen R, Bo N, Chater A, and Horne R. 2017. Beliefs 596 about medicines and non-adherence in patients with stroke, diabetes mellitus and 597 rheumatoid arthritis: a cross-sectional study in China. BMJ open 7:e017293. 10.1136/bmjopen-2017-017293

599

600

601

602

603

604 
Fig.1 A. In the Lasso model, a five-fold cross-validation approach was used for the choice of optimal parameters.Using the partial likelihood anomaly curve and the log (lambda) plot, the vertical line was drawn at the optimal value to obtain the included feature factors. $\mathrm{B}$. The lambda curve generated a profile based on the log (lambda) sequence. Vertical lines were drawn at the values selected using the five-fold cross-validation method, with 20 characteristic factors being selected. C. Algorithm of SVM-RFE support vector machine was used to further screen the 20 characteristic factors. Finally, a prediction model with 9 best features with an average 10-fold cross-validation score of 0.8914 was established.

Fig. 2 A nomogram model predicting the likelihood of benefit from surgery. Note: 9 factors including history of nasal surgery, preoperative SNOT-22 score, sinusitis, middle turbinate plasty, BMI, smoking, follow-up, advanced age, and seasonal allergies were included. $* \mathrm{p}<0.05, * * * \mathrm{p}<0.005$

Fig. 3 A.A calibration curve for the prediction model showing the benefits of endoscopic nasal septoplasty. The diagonal dashed line represents a perfect prediction of an ideal model. The solid line indicates the predictive power of the predictive model, and an improved predictive ability was observed when it closely fitted with the dotted line. B. The area under the curve (AUC) of the nomogram model indicates the probability of accurately predicting the likelihood of benefit from surgery in a randomly selected patient. The model exhibited good predictive power, with the AUC values of the training group (red), the test group (blue) and the external dataset (orange) recorded 
625 as $0.920,0.834$, and 0.765 , respectively. C. Decision curve used to estimate the surgical benefits.

626 Decision analysis curves for the training, test, and overall groups are shown. The "None" line

627 assumes that all patients failed to achieve the effect of surgery. The "All" line assumes that all 628 patients achieved the effect of surgery. 


\section{Figure 1}

In the Lasso model, a five-fold cross-validation approach was used for the choice of optimal parameters

A. In the Lasso model, a five-fold cross-validation approach was used for the choice of optimal parameters.Using the partial likelihood anomaly curve and the log (lambda) plot, the vertical line was drawn at the optimal value to obtain the included feature factors. B. The lambda curve generated a profile based on the log (lambda) sequence. Vertical lines were drawn at the values selected using the five-fold cross-validation method, with 20 characteristic factors being selected. C. Algorithm of SVM-RFE support vector machine was used to further screen the 20 characteristic factors. Finally, a prediction model with 9 best features with an average 10 -fold cross-validation score of 0.8914 was established.

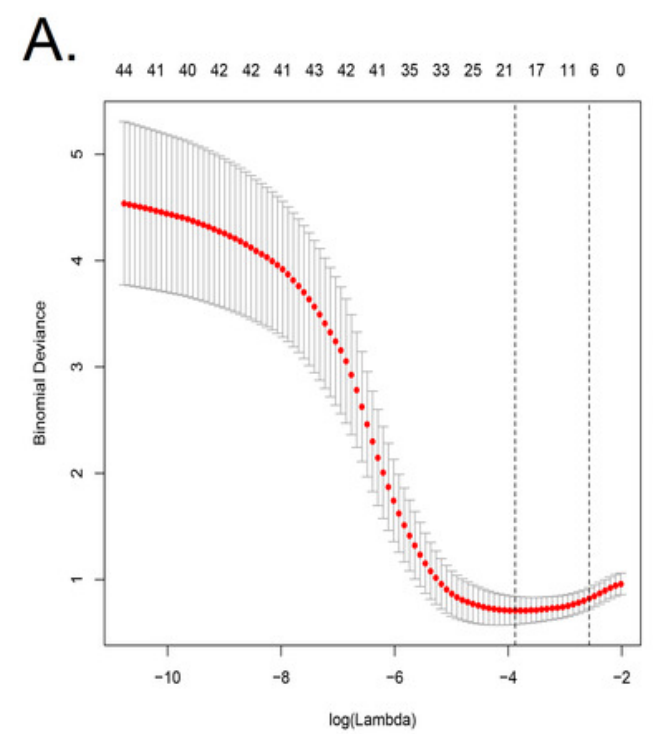

B.

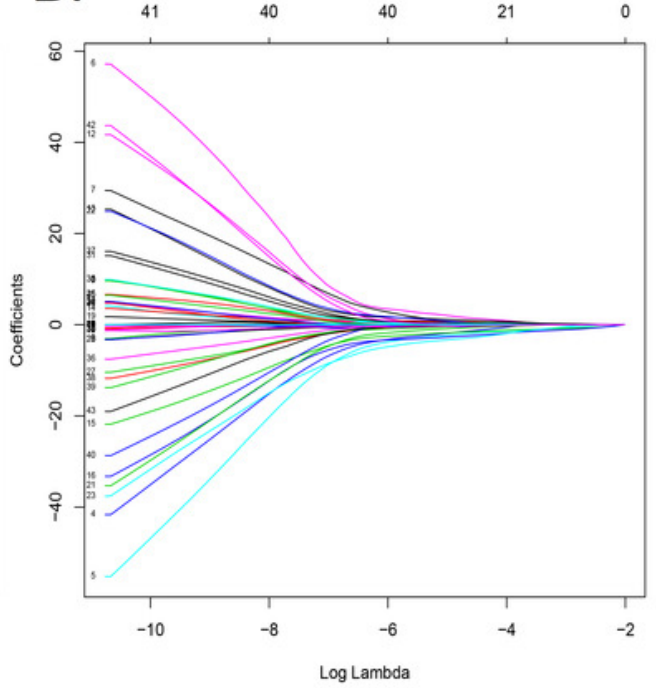

C.

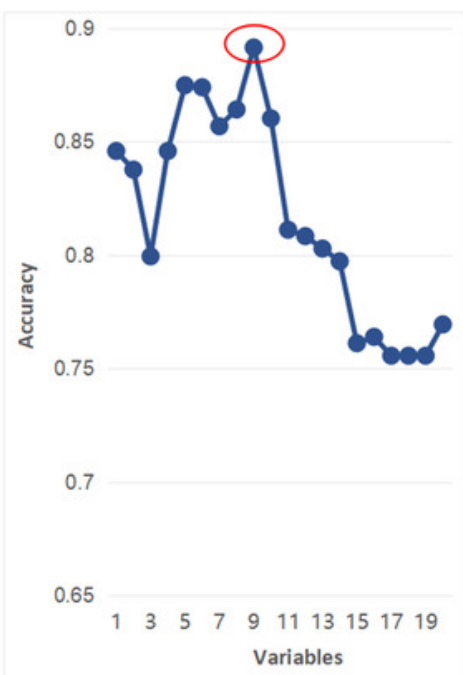


Figure 2

A nomogram model predicting the likelihood of benefit from surgery

A nomogram model predicting the likelihood of benefit from surgery. Note: 9 factors including history of nasal surgery, preoperative SNOT-22 score, sinusitis, middle turbinate plasty, BMI, smoking, follow-up, advanced age, and seasonal allergies were included. $* p<0.05, * * * p<0.005$.

BMI

smoke

middle_turbinate_plasty

Follow_up_time

seansonal_allergy_history

age $^{\star}$

SNOT22_Score*

nasosinusitis ${ }^{\star \star *}$

nasal_surgery_history ${ }^{\star \star \star}$
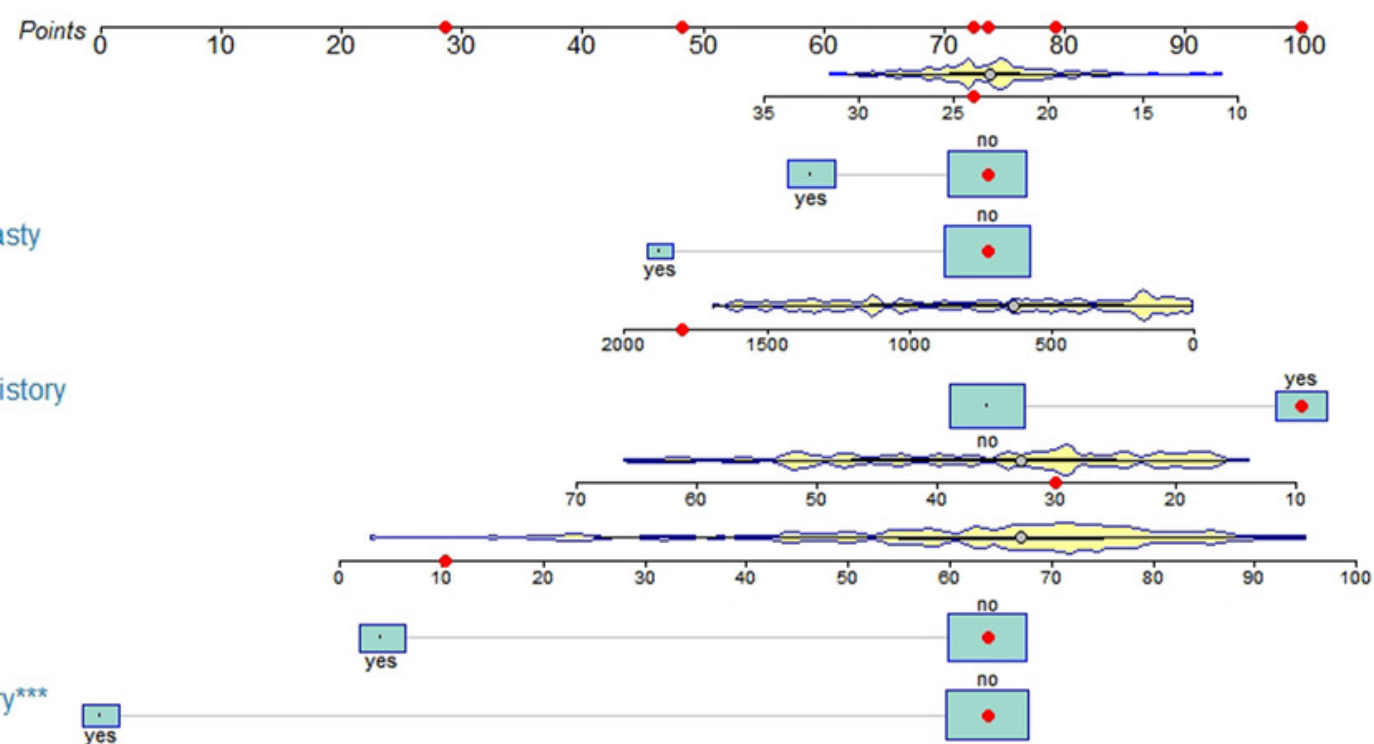

Total-points-to-outcome nomogram:

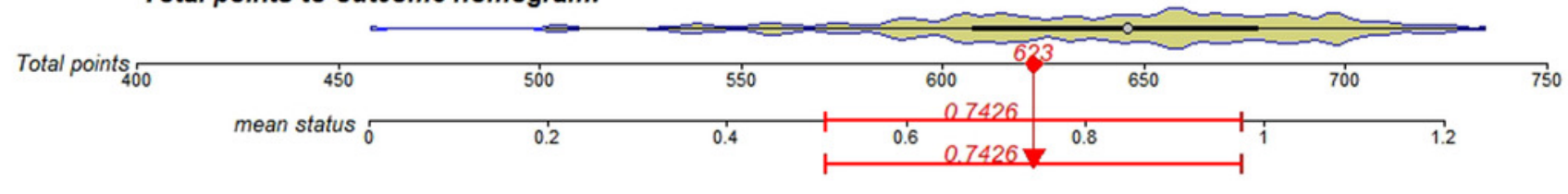




\section{Figure 3}

A calibration curve for the prediction model showing the benefits of endoscopic nasal septoplasty

A.A calibration curve for the prediction model showing the benefits of endoscopic nasal septoplasty. The diagonal dashed line represents a perfect prediction of an ideal model. The solid line indicates the predictive power of the predictive model, and an improved predictive ability was observed when it closely fitted with the dotted line. B. The area under the curve (AUC) of the nomogram model indicates the probability of accurately predicting the likelihood of benefit from surgery in a randomly selected patient. The model exhibited good predictive power, with the AUC values of the training group (red), the test group (blue) and the external dataset (orange) recorded as $0.920,0.834$, and 0.765 , respectively. C. Decision curve used to estimate the surgical benefits. Decision analysis curves for the training, test, and overall groups are shown. The "None" line assumes that all patients failed to achieve the effect of surgery. The "All" line assumes that all patients achieved the effect of surgery.

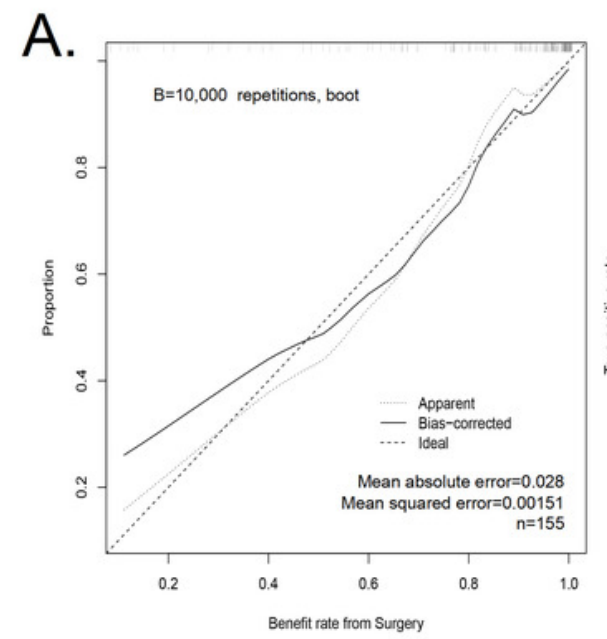

B.

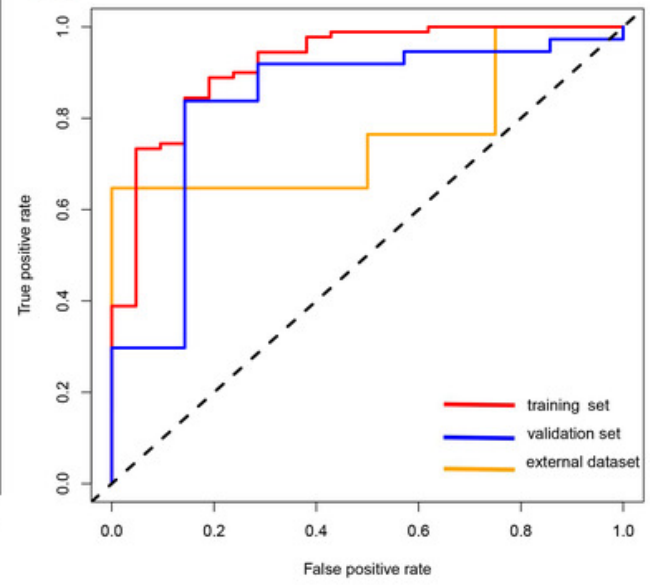

C.

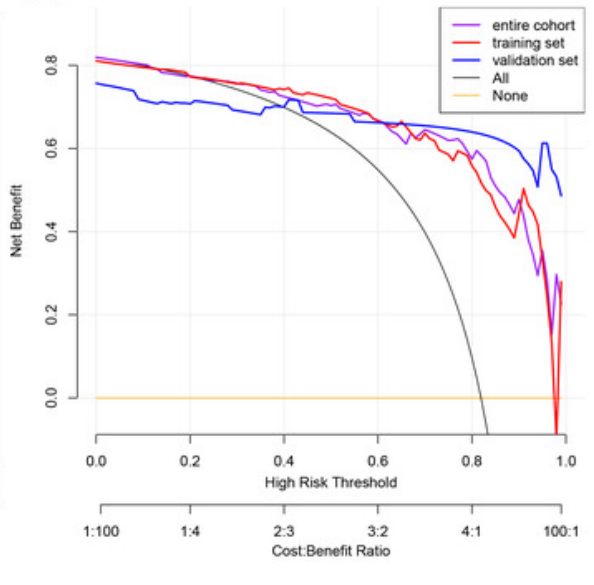




\section{Table $\mathbf{1}$ (on next page)}

Table 1 Differences of Demographic And Clinical Characteristics Between Effective And Ineffective Groups 
Table 1 Differences of Demographic And Clinical Characteristics Between Effective And Ineffective Groups

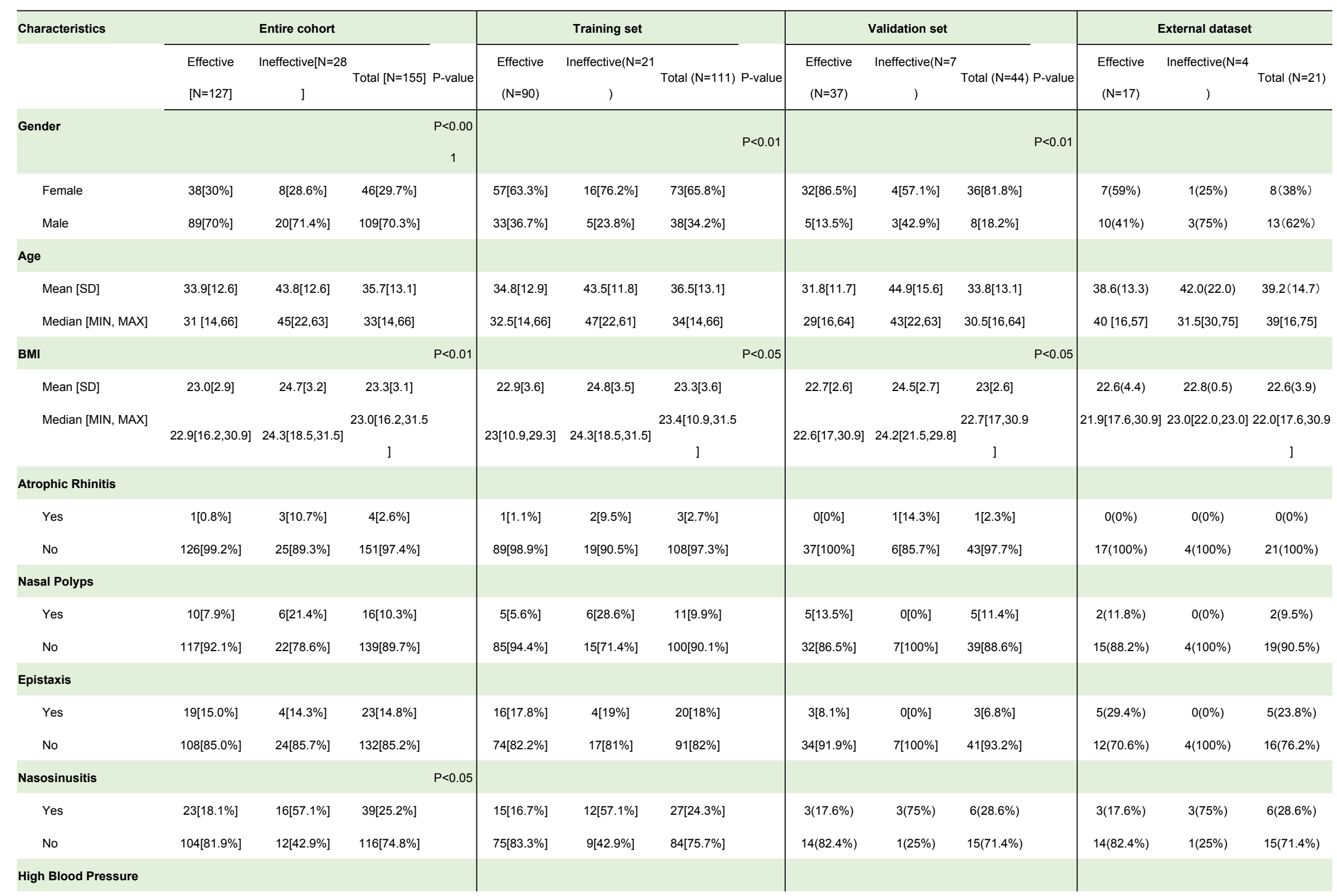




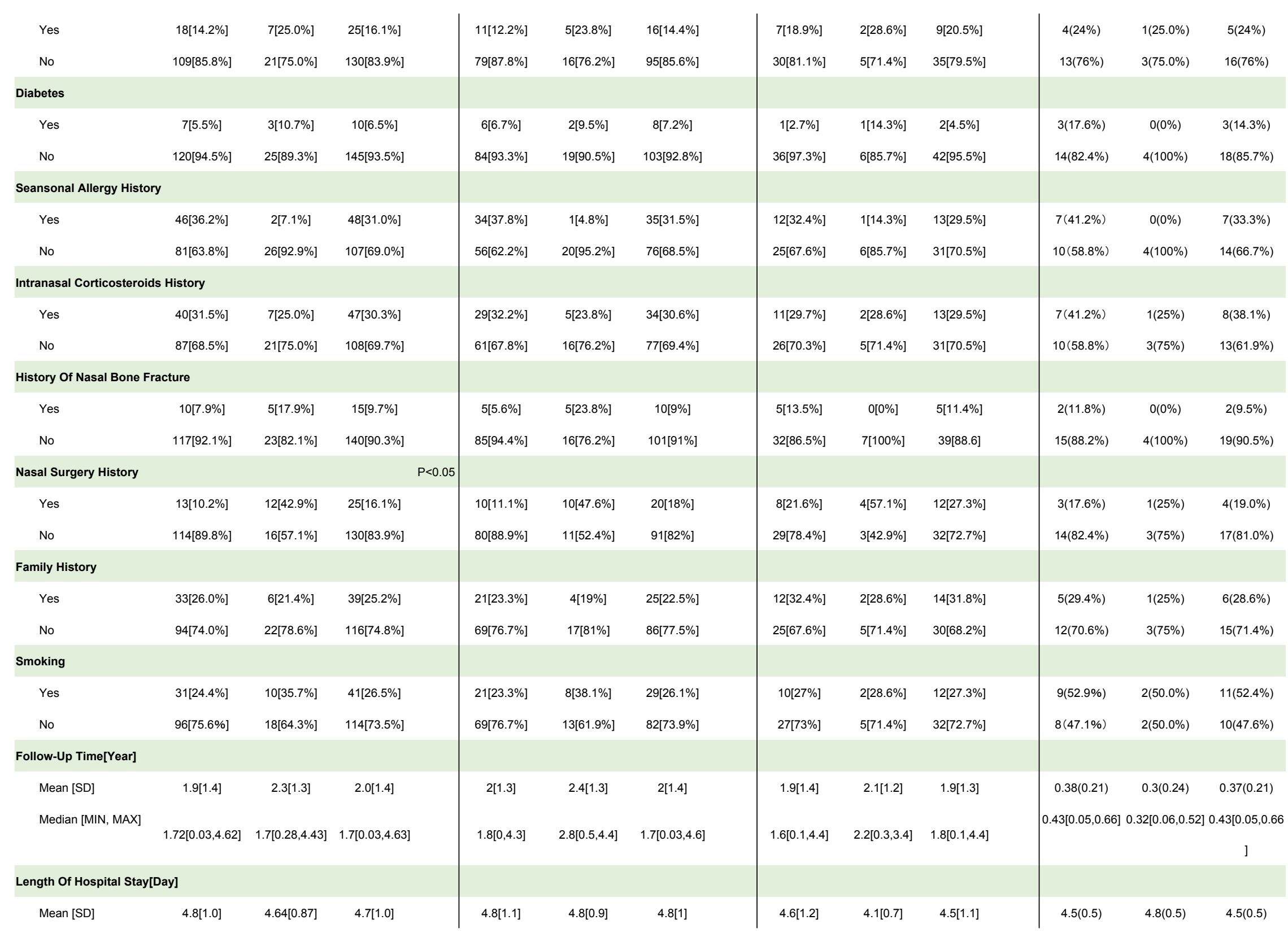




\begin{tabular}{|c|c|c|c|c|c|c|c|c|c|c|c|c|}
\hline Median [MIN, MAX] & $5[3,10]$ & $5[3,7]$ & $5[3,10]$ & $5[3,10]$ & $5[4,7]$ & $5[3,10]$ & $4[0,7]$ & $4[3,5]$ & $4[0,7]$ & $4[4,5]$ & $5[4,5]$ & $5[4,5]$ \\
\hline \multicolumn{13}{|c|}{ Proposed classification system of SD } \\
\hline Type I & $20[15.7 \%]$ & $1[3.6 \%]$ & $21[13.5 \%]$ & $12[13.3 \%]$ & $1[4.8 \%]$ & $13[11.7 \%]$ & $8[21.6 \%]$ & $0[0 \%]$ & $8[18.2 \%]$ & $2[11.8 \%]$ & $0[0 \%]$ & $2[9.5 \%]$ \\
\hline Type II & $83[65.4 \%]$ & $22[78.6 \%]$ & $105[67.7 \%]$ & $58[64.4 \%]$ & $16[76.2 \%]$ & $74[66.7 \%]$ & $25[67.6 \%]$ & $6[85.7 \%]$ & $31[70.5 \%]$ & $1[5.9 \%]$ & $2[50 \%]$ & $3[14.3 \%]$ \\
\hline Type III & $20[15.7 \%]$ & $4[14.3 \%]$ & $24[15.5 \%]$ & $18[20 \%]$ & $3[14.3 \%]$ & $21[18.9 \%]$ & $2[5.4 \%]$ & $1[14.3 \%]$ & $3[6.8 \%]$ & $9[52.9 \%]$ & $1[25 \%]$ & $10[47.6 \%]$ \\
\hline Type IV & $4[3.1 \%]$ & $1[3.6 \%]$ & $5[3.2 \%]$ & $2[2.2 \%]$ & $1[4.8 \%]$ & $3[2.7 \%]$ & $2[5.4 \%]$ & $0(0 \%)$ & $2[4.5 \%]$ & $5[29.4 \%]$ & $1[25 \%]$ & $6[28.6 \%]$ \\
\hline \multicolumn{13}{|l|}{ Additional Surgery } \\
\hline Turbinoplasty & & & & & & & & \\
\hline Yes & $11[8.7 \%]$ & $2[7.1 \%]$ & $13[8.4 \%]$ & $9[10 \%]$ & $1[4.8 \%]$ & $10[9 \%]$ & $2[5.4 \%]$ & $1[14.3 \%]$ & $3[6.8 \%]$ & $2(11.8 \%)$ & $0(0 \%)$ & $2(9.5 \%)$ \\
\hline No & $116[91.3 \%]$ & $26[92.9 \%]$ & 142[91.6\%] & $81[90 \%]$ & $20[95.2 \%]$ & 101[91\%] & $35[94.6 \%]$ & $6[85.7 \%]$ & $41[93.2 \%]$ & $15(88.2 \%)$ & $4(100 \%)$ & $19(90.5 \%)$ \\
\hline \multirow{2}{*}{\multicolumn{13}{|c|}{$\begin{array}{l}\text { Inferior } \\
\text { Turbinoplasty }\end{array}$}} \\
\hline & & & & & & & & & & & & \\
\hline Yes & $58[45.7 \%]$ & $10[35.7 \%]$ & $68[43.9 \%]$ & $40[44.4 \%]$ & $8[38.1 \%]$ & $48[43.2 \%]$ & $18[48.6 \%]$ & $2[28.6 \%]$ & $20[45.5 \%]$ & $7(41.2 \%)$ & $4(100 \%)$ & $11(52.4 \%)$ \\
\hline No & $69[54.3 \%]$ & $18[64.3 \%]$ & $87[56.1 \%]$ & $50[55.6 \%]$ & $13[61.9 \%]$ & $63[56.8 \%]$ & $19[51.4 \%]$ & $5[71.4 \%]$ & $24[54.5 \%]$ & $10(58.8 \%)$ & 0(0\%) & $10(47.6 \%)$ \\
\hline \multicolumn{13}{|l|}{ Augmentation } \\
\hline \multicolumn{13}{|l|}{ Rhinoplasty } \\
\hline Yes & $8[6.2 \%]$ & $0[0 \%]$ & $8[5.2 \%]$ & $6[6.7 \%]$ & $0[0 \%]$ & $6[5.4 \%]$ & $2[5.4 \%]$ & $0[0 \%]$ & $2[4.5 \%]$ & $3(17.6 \%)$ & $0(0 \%)$ & $3(14.3 \%)$ \\
\hline No & $119[85.8 \%]$ & $28[100 \%]$ & $147[94.8 \%]$ & $84[93.3 \%]$ & $21[100 \%]$ & 105[94.6\%] & $35[94.6 \%]$ & $7[100 \%]$ & $42[95.5 \%]$ & $14(82.4 \%)$ & $4(100 \%)$ & $18(85.7 \%)$ \\
\hline \multicolumn{13}{|l|}{ RFVR Of The Inferior } \\
\hline \multicolumn{13}{|l|}{ Turbinate } \\
\hline Yes & $8[6.2 \%]$ & $1[3.6 \%]$ & $9[5.8 \%]$ & $5[5.6 \%]$ & $0[0 \%]$ & $5[4.5 \%]$ & $3[8.1 \%]$ & $1[14.3 \%]$ & $4[9.1 \%]$ & $1(5.9 \%)$ & $1(25 \%)$ & $2(9.5 \%)$ \\
\hline No & $109[85.8 \%]$ & $27[96.4 \%]$ & $146[94.2 \%]$ & $85[94.4 \%]$ & $21[100 \%]$ & $106[95.5 \%]$ & $34[91.9 \%]$ & $6[85.7 \%]$ & $40[90.9 \%]$ & $16(94.1 \%)$ & $3(75 \%)$ & $19(90.5 \%)$ \\
\hline \multicolumn{13}{|l|}{ Nasal Bone Fracture } \\
\hline \multicolumn{13}{|l|}{ Reduction } \\
\hline Yes & $4[3.1 \%]$ & $1[3.6 \%]$ & $5[3.2 \%]$ & $2[2.2 \%]$ & $1[4.8 \%]$ & $3[2.7 \%]$ & $2[5.4 \%]$ & $0[0 \%]$ & $2[4.5 \%]$ & $3(17.6 \%)$ & $0(0 \%)$ & $3(14.3 \%)$ \\
\hline No & $123[96.9 \%]$ & $27[96.4 \%]$ & $150[96.8 \%]$ & $88[97.8 \%]$ & $20[95.2 \%]$ & 108[97.3\%] & $35[94.6 \%]$ & $7[100 \%]$ & $42[95.5 \%]$ & $14(82.4 \%)$ & $4(100 \%)$ & $18(85.7 \%)$ \\
\hline \multicolumn{13}{|l|}{ Endoscopic Sinus } \\
\hline Surgery & & & & & & & & & & & & \\
\hline
\end{tabular}




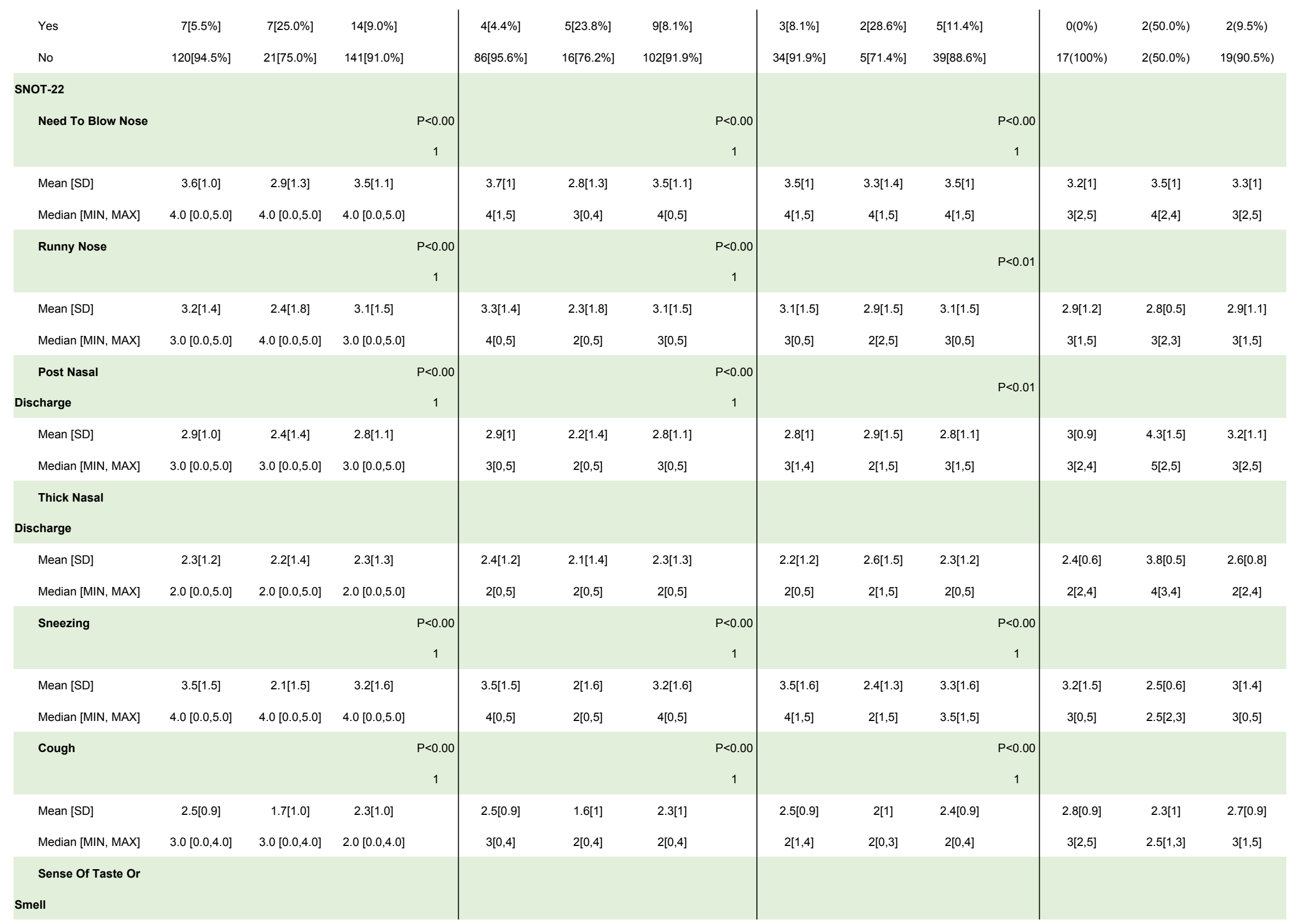




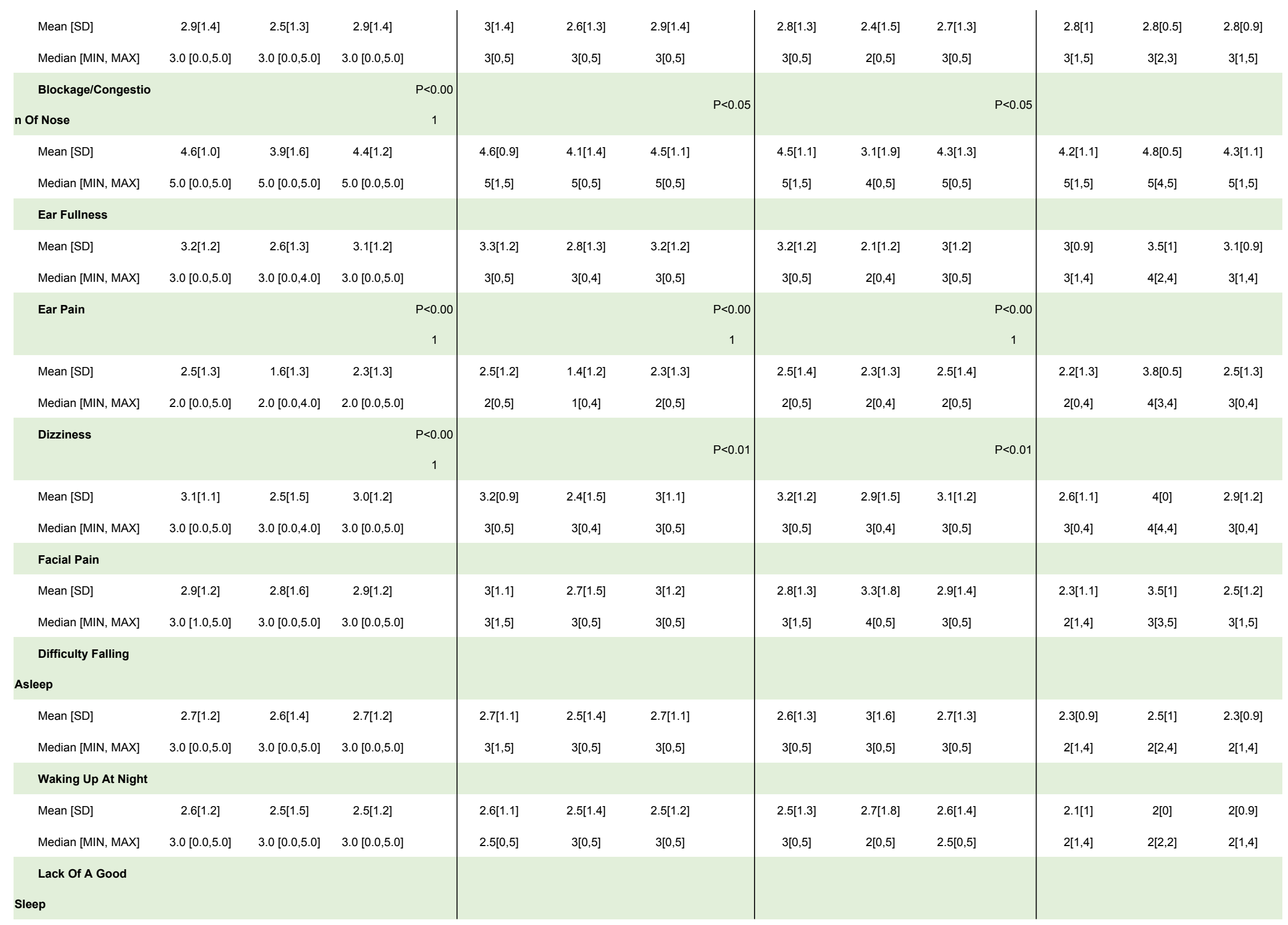




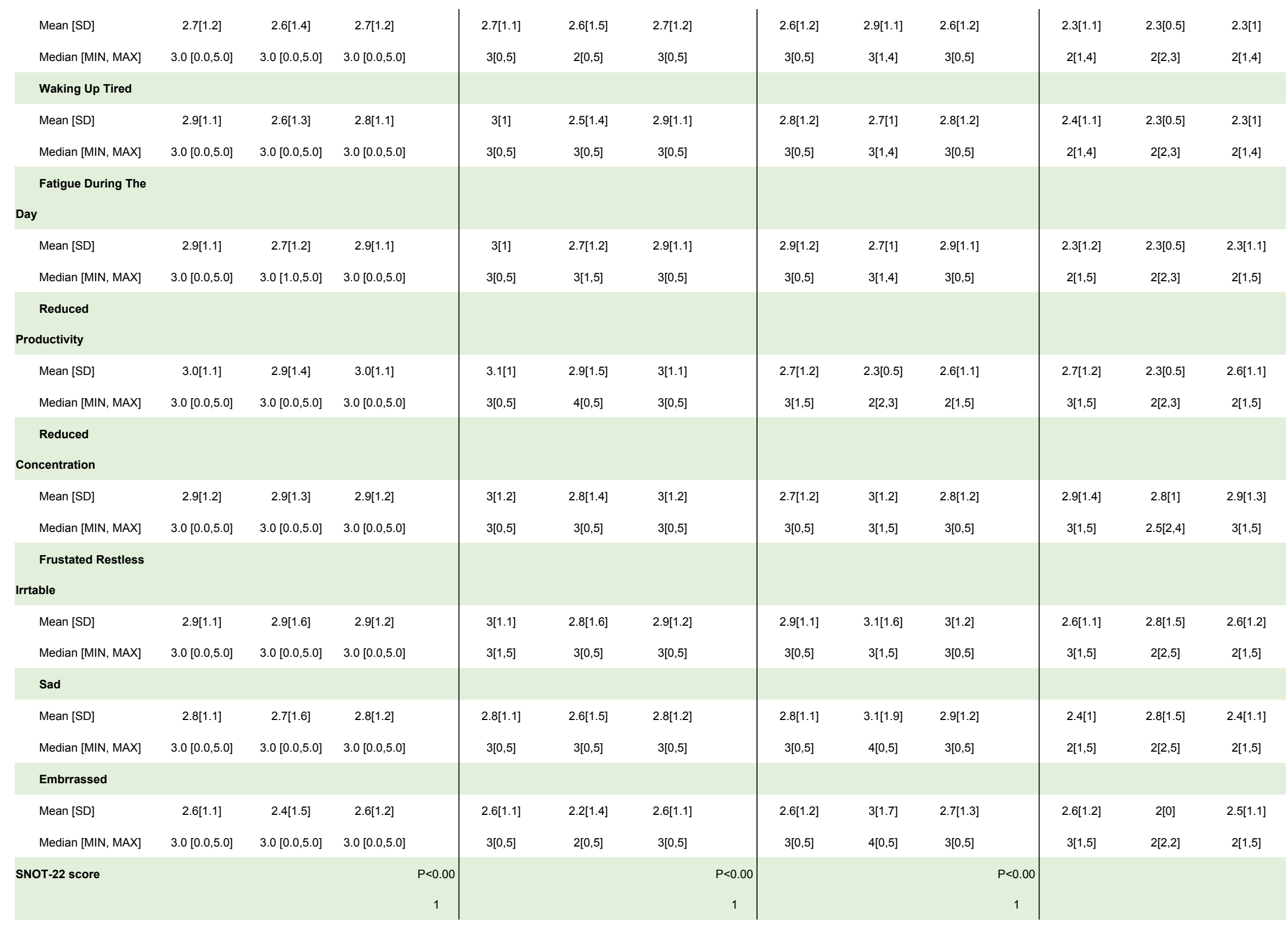


PeerJ

Manuscript to be reviewed

\begin{tabular}{|c|c|c|c|c|c|c|c|c|c|c|c|}
\hline Mean [SD] & $65.3[16.4]$ & $63.7[17.4]$ & $66.3[14.6]$ & $54.7[19.6]$ & $64.1[16.2]$ & $63.9[17.9]$ & $62.9[18.3]$ & $63.7[17.8]$ & $59.1[14.7]$ & $65[1.6]$ & $60.2[13.3]$ \\
\hline Median [MIN, MAX] & $68.0[3.0,95.0]$ & $69.0[15.0,83.0] 67.0[3.0,95.0]$ & $69[19,95]$ & $62[23,83]$ & $67[19,95]$ & $67[21,93]$ & $71[25,78]$ & $67.5[21,93]$ & $59[35,76]$ & $65[63,67]$ & $65[35,76]$ \\
\hline
\end{tabular}


Table 2 (on next page)

Table 2 Rank the order of features in SVM-RFE method 
Table 2 Rank the order of features in SVM-RFE method

\begin{tabular}{lcc}
\hline Features & Coefficients & Rank the order \\
\hline Middle turbinate plasty & -4.75266432 & 1 \\
Nasosinusitis & -2.21413383 & 2 \\
Nasal surgery history & -2.3517654 & 3 \\
Seansonal allergy history & 3.03876115 & 4 \\
Age & -0.06223234 & 5 \\
SNOT22 Score & 0.03536382 & 6 \\
BMI & -0.17801399 & 7 \\
Smoke & -1.088097 & 8 \\
Follow up time & -0.35657536 & 9 \\
\hline
\end{tabular}

1 
Table 3 (on next page)

Table 3 Chart of prediction factors 
Table 3 Chart of prediction factors

\begin{tabular}{|c|c|c|c|c|}
\hline \multirow[t]{2}{*}{ Variable } & \multicolumn{4}{|c|}{ Prediction model } \\
\hline & $\beta$ & & Odds ratio $(95 \% \mathrm{Cl})$ & P-value \\
\hline SNOT22 score & & 0.034 & $1.035(1.004-1.070)$ & 0.028 \\
\hline Age & & -0.059 & $0.943(0.895-0.988)$ & 0.019 \\
\hline Smoke & & -0.678 & $0.508(0.154-1.680)$ & 0.259 \\
\hline Seansonal allergy history & & 2.189 & 8.930(1.817-74.700) & 0.017 \\
\hline Nasosinusitis & & -2.003 & $0.135(0.034-0.460)$ & 0.002 \\
\hline Follow up time & & -0.001 & 0.999(0.998-1.000) & 0.043 \\
\hline BMI & & -0.065 & $0.937(0.780-1.110)$ & 0.471 \\
\hline Nasal surgery history & & -2.823 & $0.059(0.013-0.227)$ & 0.0000975 \\
\hline Middle turbinate plasty & & -1.875 & $0.153(0.017-1.800)$ & 0.104 \\
\hline
\end{tabular}

Note: $\beta$ is the regression coefficient.

1 